\title{
Estado nutricional de idosos atendidos na atenção primária à saúde do município de Candelária/RS
}

\author{
Nutritional status of elderly patients in primary health \\ care in the municipality of Candelaria/RS
}

\begin{abstract}
Bianca Bernhard dos Santos, ${ }^{1}$ Analie Nunes Couto, ${ }^{1}$ Francisca Maria Assmann Wichmann ${ }^{1}$ 'Universidade de Santa Cruz do Sul (Unisc), Santa Cruz do Sul, RS, Brasil.
\end{abstract}

Recebido em: 21/08/2016 / Aceito em: 29/09/2016 / Publicado em: 18/10/2016

francis@unisc.br

\section{RESUMO}

Objetivo: descrever o perfil nutricional de idosos atendidos na Atenção Primária à Saúde do município de Candelária/RS. Método: trata-se de um estudo retrospectivo, de natureza descritiva com delineamento transversal e abordagem quantitativa, constituída por uma amostra aleatória de 247 idosos com idade superior a 60 anos, de ambos os gêneros, residentes em Candelária, no Rio Grande do Sul que buscaram tratamento e cuidado de saúde nos serviços de saúde do município. As seguintes variáveis foram analisadas: idade, gênero, escolaridade, peso, estatura, índice de massa corporal, comorbidades e estado nutricional, sendo este último analisado com base no índice de massa corporal (IMC) calculado com base na divisão do peso corporal em quilogramas pela estatura em metro elevada ao quadrado $\left(\mathrm{kg} / \mathrm{m}^{2}\right)$. Resultados: a amostra foi constituída por $59,9 \%(n=148)$ idosos do sexo feminino e $40,1 \%(n=99)$ do sexo masculino, com a média da idade de $75,25 \pm 6,426$. Em relação ao estado nutricional, verificou-se uma prevalência significativa do sexo feminino com pré-obesidade e obesidade na faixa etária de 60 a 79 anos e no sexo masculino com idade maior ou igual a 80 anos. Verificou-se forte associação entre o estado nutricional determinado pelo IMC à HAS e a baixa escolaridade. Considerações finais: os dados relativos ao IMC revelaram altos percentuais de excesso de peso, o que suscita a necessidade de outros métodos complementares de avaliação para identificação mais precisa dos fatores de risco e dos agravantes que se associam as patologias encontradas.

Palavras-chave: Estado nutricional; Idoso; Atenção Primária à Saúde; Doença crônica não transmissível; Saúde do idoso.

\section{ABSTRACT}

Objective: describe the nutritional status of elderly assisted in primary health care in the municipality of Candelaria/RS. Method: this is a retrospective study of descriptive cross-sectional design and quantitative approach, consisting of a random sample of 247 elderly aged 60 years, of both genders, residents in Candelaria, in Rio Grande do Sul that sought treatment and health care at the municipal health services. The following variables were analyzed: age, gender, education, weight, height, body mass index, comorbidities and nutritional status, the latter being analyzed based on body mass index (BMI) calculated by dividing body weight in kilograms by the elevated height in meters squared $\left(\mathrm{kg} / \mathrm{m}^{2}\right)$. Results: sample consisted of $59.9 \%$ ( $n=148$ ) female elderly and $40.1 \%$ ( $n=99)$ male, with a mean age of $75.25 \pm 6.426$. In relation to nutritional status, there was a significant prevalence of women with overweight and obesity in the age group 60-79 years and males aged greater than or equal to 80 years. There was a strong association between nutritional status determined by BMI to high blood pressure (HBP) and low education. Closing remarks: the data on BMI showed high percentages of overweight, which raises the need for other complementary assessment methods for more accurate identification of risk factors and aggravating factors that are associated pathologies found.

Keywords: Nutritional status; Elderly; Primary Health Care; Not transmissible chronic disease; Elderly Health. 


\section{INTRODUÇÃO}

O envelhecimento da população é uma realidade global. A Organização Mundial de Saúde (OMS) prevê que em 2025 existirão 1,2 bilhões de pessoas com mais de 60 anos no mundo, 34 milhões delas no Brasil. Estimativas apontam que a proporção de idosos com 65 anos ou mais são os que mais estão crescendo ${ }^{1}$ e recentemente pesquisas apontam um número crescente de idosos mais longevos (com idade acima de 80 anos), sendo que estes grupos terão pelo menos uma Doença Crônica Não Transmissível - DCNT. ${ }^{2}$

Viver mais, não significa viver melhor, pois na medida em que a expectativa de vida aumenta, se elevam os fatores de risco para as doenças crônicas-degenerativas e suas complicações. A maioria das doenças crônicas que acometem o idoso tem na própria idade seu principal fator de risco. Além do mais, observa-se que a redução nos índices da mortalidade vem acompanhada com um número expressivo de idosos longevos que apresentam deficiências clínicas e doenças crônicas e estes aumentam a demanda nos serviços de saúde. ${ }^{3}$

Ao considerar que os idosos apresentam características advindas da idade que podem comprometer seu estado nutricional, é importante monitorar a situação nutricional dos idoso ${ }^{4}$ A OMS propõe fortalecer o nível primário de atenção à saúde do idoso e desenvolver indicadores capazes de avaliar o estado de saúde, a presença de doenças crônicas e o estado nutricional, sendo que este último pode ser inclusive, um indicador positivo de saúde. ${ }^{1}$

Cabe salientar, que a elevada prevalência de desvios nutricionais na população idosa, vem sendo demonstrada por meio de diferentes estudos, em vários países, onde a desnutrição, o sobrepeso e a obesidade predominam sobre os indivíduos eutróficos. Estes distúrbios nutricionais fazem com que os idosos se tornem susceptíveis a ocorrência de doenças crônicas, redução da capacidade funcional e maior mortalidade. ${ }^{5}$

Diante do exposto, o panorama da saúde nutricional do idoso precisa ser, sem dúvida, melhor definido, com levantamentos antropométricos populacionais periódicos e da coleta sistemática e contínua de dados provenientes dos serviços de saúde para que se possa estabelecer práticas de monitoramento nutricional e direcionar intervenções mais adequadas. ${ }^{4,6}$ Este estudo teve como objetivo descrever o perfil nutricional de idosos atendidos na Atenção Primária à Saúde do município de Candelária/RS.

\section{MÉTODO}

Trata-se de um estudo retrospectivo, de natureza descritiva com delineamento transversal e abordagem quantitativa. A população foi constituída por uma amostra aleatória de idosos ( $\geq 60$ anos de idade), residentes em Candelária, Rio Grande do Sul e que buscaram tratamento e cuidados de saúde nos serviços de saúde de Candelária-RS, durante o ano de 2015. A amostragem foi realizada de forma aleatória simples. O estudo foi aprovado pelo Comitê de Ética em Pesquisa da instituição sob o protocolo $n^{\circ} 553501$.

As informações relativas à pesquisa foram extraídas dos prontuários eletrônicos dos idosos. A coleta dos dados ocorreu entre os meses de abril e maio de 2016. Utilizou-se um formulário com questões sócio de- mográficos, condições de saúde, perfil antropométrico. As seguintes variáveis foram analisadas: idade, gênero, escolaridade, peso, estatura, índice de massa corporal, comorbidades e estado nutricional.

A variável dependente analisada foi o índice de massa corporal (IMC) calculado com base na divisão do peso corporal em quilogramas pela estatura em metro elevada ao quadrado $\left(\mathrm{kg} / \mathrm{m}^{2}\right)$. O estado nutricional dos idosos foi avaliado, segundo pontos de corte propostos pela Organização Pan-Americana da Saúde7: baixo peso (IMC $\left.<23 \mathrm{~kg} / \mathrm{m}^{2}\right)$, peso normal $\left(23<\mathrm{IMC}<28 \mathrm{~kg} / \mathrm{m}^{2}\right)$, pré-obesidade $\left(28 \leq \mathrm{IMC}<30 \mathrm{~kg} / \mathrm{m}^{2}\right)$ e obesidade (IMC $>30 \mathrm{~kg}$ / $\mathrm{m}^{2}$ ). Os pacientes foram divididos em duas categorias: idosos ( $\geq 60$ e $<79$ anos) e muito idosos ( $\geq 80$ anos).

As variáveis independentes analisadas foram: idade (60- 79 anos e 80 anos e mais), sexo (masculino, feminino), escolaridade (analfabeto, $1^{\circ}$ grau incompleto, $2^{\circ}$ grau incompleto e $2^{\circ}$ grau completo), massa corporal (kg), estatura $(m)$ e doenças instaladas. Estes obtidos a partir dos prontuários eletrônicos no banco de dados eletrônicos e analisados pela pesquisadora de forma a evitar vieses.

Os dados secundários foram submetidos a testes estatísticos, sendo utilizado o programa SPSS versão 20.0. Primeiramente, foi realizada análise descritiva para caracterização da amostra, em seguida o teste $T$ de Student foi empregado para verificar as diferenças entre as variáveis e o teste não paramétrico qui-quadrado, tendo como variável dependente o estado nutricional e, como variáveis independentes, sexo, escolaridade e as condições de saúde dos idosos pesquisados. O nível de significância adotado para o estudo foi de $5 \%$.

\section{RESULTADOS}

A amostra foi constituída por 247 idosos, sendo $59,9 \%(n=148)$ do sexo feminino e $40,1 \%(n=99)$ do sexo masculino. Verificou-se que a média da idade \pm desvio padrão foi de $75,25 \pm 6,426$, sendo $75,33 \pm 6,63$ anos para mulheres e $74,89 \pm 5,66$ para homens. Quanto à escolaridade, verifica-se que $8,1 \%$ dos idosos que buscam atendimento em saúde são analfabetos. Em relação às outras categorias analisadas, o primeiro grau incompleto aparece como a mais prevalente, correspondendo a $57,5 \%$.

Os registros clínicos mais prevalentes identificados nos prontuários eletrônicos, considerando ambos os sexos, correspondem a HAS associada à DM $(65,6 \%)$. No que se refere às morbidades com associação significativa de pré-obesidade, identificou-se que, dentre os idosos portadores de HAS, 55\% estão com pré-obesidade $(p=0,04)$. Em relação às doenças mais prevalentes o estudo revela que a HAS se mantém alta entre os dois sexos com altas taxas de pré-obesidade.

Em referência à escolaridade e o estado nutricional houve uma associação significativa $(p=0,034)$. Em relação às medidas antropométricas observou-se que a média do peso foi de $74,21 \pm 15,47$, sendo $75,33 \pm 6,632$ para mulheres e $74,50 \pm 17,25$ para homens. Verificou-se uma prevalência significativa de obesidade $(38,9 \%)$ e pré-obesidade $(14,6 \%)$ entre os idosos avaliados. Em relação ao baixo peso houve uma prevalência maior no sexo masculino $(18,2 \%)$ quando comparado ao sexo feminino $(8,2 \%)$. Do mesmo modo em o estudo mostra uma prevalência maior de peso normal no sexo feminino em idades mais avançadas.

Quando analisados em relação ao sexo houve uma 
prevalência maior no sexo feminino de pré-obesidade e obesidade na faixa etária de 60 a 79 anos e no sexo masculino com idade maior ou igual a 80 anos. Quanto à análise estatística houve uma associação significativa entre o Índice de Massa Corporal e a idade dos idosos $(p=0,00)$; não houve diferença entre os sexos em relação ao estado nutricional $(p=0,17)$. No sexo feminino, $o$ percentual de pré-obesidade foi de $54,4 \%(n=81)$ do total da amostra, com média de IMC $28,67 \pm 5,84 \mathrm{Kg} / \mathrm{m}^{2}$. No masculino, o percentual de sobrepeso foi de $51,1 \%$ ( $n=51$ ), com média de IMC 29,50 55,71 (Tabela 1).

Ao analisar os serviços de saúde, a unidade de saúde Marilene e a Unidade Básica Central possuem a maior prevalência de pré-obesidade e obesidade, seguidas das unidades Irene de Silva Oliveira e Rincão. Quanto à análise estatística não houve uma associação significativa em relação às faixas etárias as unidades e o estado nutricional da amostra estudada $(p=0,97)$. Os dados produzidos na rotina do Serviço de Saúde representam a população idosa do município e pode-se perceber, pelos resultados encontrados, a importância do monitoramento do estado nutricional deste grupo populacional.

\section{DISCUSSÃO}

O presente estudo teve sua amostra constituída por $89 \%$ com idade entre 60- 79 anos e $11 \%$ com idade acima de 80 anos, prevalecendo o sexo feminino $(59,9 \%)$. Esses aspectos encontrados no estudo vão ao encontro dos dados apresentados pelo IBGE $^{8}$ em relação à população idosa no Brasil, que em 2015 atingiu $11,47 \%$ da população, sendo a maioria de mulheres com $6,54 \%$ contra $5,2 \%$ de homens. No Rio Grande do Sul, os idosos representam $15,63 \%$ da população do Estado, sendo também a maioria de mulheres com $8,83 \%$ contra apenas $6,8 \%$ de homens.

As DCNT mais frequentes encontradas entre esses idosos, em ambos os sexos, são a HAS $(65,6 \%)$, HAS associada à DM e depressão (16,6\%). No estudo de Vasconcelos et al. ${ }^{3}$ quanto às morbidades associadas ao excesso de peso, houve predominância da HAS, $68,2 \%$, seguida das dislipidemias $(55,6 \%)$ e das doenças do aparelho musculoesquelético $(54,7 \%)$. Já no estudo de Silva et al. ${ }^{6}$ no que diz respeito à presença referida de agravos, encontrou-se $54,7 \%$ de HAS.

Dessa forma, é possível considerar resultados que se assemelham aos encontrados neste estudo em relação às DCNT mais frequentes, pois em uma pesquisa apresentada por Cavalcanti et. al., ${ }^{9}$ sobre a prevalência de doenças crônicas e estado nutricional em um grupo de idosos brasileiros, as patologias mais frequentes encontradas foram a HAS com 56,4\%, e a DM, com $20,5 \%$, sendo o mesmo diagnóstico encontrado nos estudos de Bassler e Lei $^{10}$ sendo HAS $(59,8 \%)$ e DM $(15,5 \%)$, e na pesquisa apresentada por Alves et al., ${ }^{11}$ onde a HAS foi a patologia crônica mais frequente com $53,4 \%$ de acometimento em idosos.

Em relação ao estado nutricional, com base nas medidas antropométricas observou-se uma prevalência significativa de IMC pré-obesidade $(29,17 \pm 5,77 \mathrm{Kg} /$ $\mathrm{m}^{2}$ ), porém não se constatou uma grande diferença entre homens $51,1 \%(n=51)$ e mulheres $54,4 \%(n=81)$. 0 estudo aponta uma prevalência de $12 \%(n=) 31$ de baixo peso entre os idosos, sendo que $14,8 \%(n=4)$ em idosos longevos ( $\geq 80$ anos) e 12,3\% ( $n=27)$ entre 60 e 79 anos, sem diferença significante entre as faixas etárias.

De acordo com os dados levantados, em relação ao grupo geral de idosos, ou seja, de ambos os sexos, a pesquisa apresentada por Silva et al. ${ }^{6}$ também demonstrou que a maioria dos idosos possuía excesso de peso chegando a um percentual de $83,3 \%$. Da mesma forma, a pesquisa apresentada por Scherer et al. ${ }^{12}$ sobre o estado nutricional e a prevalência de doenças crônicas em idosos também constatou que o excesso de peso é maior nas mulheres idosas $(62,5 \%)$ do que em homens idosos $(60,3 \%)$ de acordo com o IMC, 53,6\% dos idosos foram classificados com excesso de peso.

Com relação ao baixo peso, o presente estudo constatou que houve uma prevalência maior no sexo masculino $(18,2 \%)$ quando comparado ao sexo feminino $(8,2 \%)$. Neste sentido, corrobora os resultados apresentados na pesquisa de Nascimento et al. ${ }^{13}$ sobre estado nutricional e fatores associados em idosos de Viçosa/ MG, onde também foi encontrada uma prevalência de

Tabela 1 - Distribuição percentual de estados nutricionais, segundo idade, sexo e Unidades de Saúde de referência dos idosos do município de Candelária/RS.

\begin{tabular}{|c|c|c|c|c|c|c|}
\hline Total & Baixo peso & Peso Normal & Pré-obesidade & Obesidade $n$ & Total & $p^{*}$ \\
\hline & n (\%) & n (\%) & n (\%) & $(\%)$ & n (\%) & \\
\hline $60-79$ & $27(10,9)$ & $79(31,2)$ & $28(11,3)$ & $86(34,8)$ & 220 (89) & \\
\hline$>80$ & $4(1,6)$ & $6(2,4)$ & $9(3,6)$ & $10(4,1)$ & $27(11)$ & 0,09 \\
\hline Total & $31(12,5)$ & $83(33,6)$ & $37(14,9)$ & $96(38,9)$ & 247 (100) & \\
\hline \multicolumn{7}{|l|}{ Masculino } \\
\hline $60-79$ & $16(16,1)$ & $19(19,3)$ & $47(48,1)$ & $7(7)$ & $89(36)$ & \\
\hline$>80$ & $2(2,1)$ & $2(2,1)$ & $3(3)$ & $2(2)$ & $10(4)$ & 0,01 \\
\hline Total & $18(18,2)$ & $21(21,4)$ & $51(51,1)$ & $9(9,1)$ & $99(40)$ & \\
\hline \multicolumn{7}{|l|}{ Feminino } \\
\hline $60-79$ & $9(6,1)$ & $20(13,5)$ & $74(49,5)$ & $28(18,9)$ & 131 (53) & \\
\hline$>80$ & $4(2,7)$ & $2(1,3)$ & $8(5,2)$ & $3(2)$ & $17(6,9)$ & 0,01 \\
\hline Tota & $13(8,2)$ & $23(15,5)$ & $81(54,7)$ & $31(20,9)$ & $148(59,9)$ & \\
\hline \multicolumn{7}{|l|}{ ESF } \\
\hline Irene & $5(2)$ & $12(4,8)$ & $5(2)$ & $15(6)$ & $37(14,9)$ & \\
\hline Marilene & $3(1,2)$ & $10(4)$ & $6(2,4)$ & $11(4,4)$ & $30(12,2)$ & \\
\hline Rincão & $11(4,4)$ & $37(14,9)$ & $14(5,7)$ & $33(13,3)$ & $95(38,5)$ & 0,97 \\
\hline Central & $12(4,8)$ & $27(10,9)$ & $10(4)$ & $36(14,5)$ & $85(34,3)$ & \\
\hline Total & $31(12,5)$ & $86(34,8)$ & $35(14,2)$ & $95(38,9)$ & $247(100)$ & \\
\hline
\end{tabular}

* Teste qui-quadrado 
baixo peso significante maior entre os homens $(18,2 \%)$ se comparada às mulheres $(9,0 \%)$, do mesmo modo em que refere o índice de massa corporal diminui com a idade em ambos os sexos. França et al. ${ }^{4}$ detectou a condição nutricional de baixo peso segundo o IMC mais prevalente entre idosos do sexo masculino com idade mais avançada.

O IMC é bastante utilizado em virtude de sua fácil aplicação e baixo custo, entretanto não reflete a redistribuição regional de gordura, devendo ser associado a outros métodos de avaliação. O resultado de sobrepeso e obesidade torna-se preocupante devido a algumas doenças comumente serem potencializadas pela obesidade, assumindo maior importância entre os indivíduos idosos, pois aumentam a morbi-mortalidade e diminuem a qualidade de vida dos idosos, causando um impacto sobre o sistema de saúde..$^{14}$

Os idosos requerem maiores demandas de assistência para o tratamento de doenças crônicas e deficiências, por apresentarem mais desigualdades em saúde, devido a circunstâncias sociais e econômicas determinadas por condições de vida e trabalho desiguais..$^{15}$ Mediante a isso, a atenção à saúde dos idosos deve voltar-se para estratégias de monitorização e de planejamento pelos serviços que atendem esta população, a fim de prevenir ou atenuar os riscos relativos aos problemas emocionais, físicos e funcionais e possibilitar uma vida mais saudável. ${ }^{16}$

\section{CONSIDERACÕEES FINAIS}

A realização deste estudo permitiu evidenciar o perfil do estado nutricional de uma população idosa atendida pela atenção básica de um município de pequeno porte. Apontou a predominância do sexo feminino na amostra e prevalência do sexo feminino com pré-obesidade e obesidade na faixa etária de 60 a 79 anos e no sexo masculino com idade maior ou igual a 80 anos. Verificou-se forte associação entre o estado nutricional determinado pelo IMC à HAS e a baixa escolaridade. Os dados relativos ao IMC revelaram altos percentuais de excesso de peso, o que suscita a necessidade de outros métodos complementares de avaliação, para identificação mais precisa dos fatores de risco e dos agravantes que se associam as patologias encontradas.

Dessa maneira, considerando-se a complexidade da influência desses fatores, se faz necessário o estabelecimento de práticas de monitoramento e o planejamento de intervenções mais direcionadas às necessidades dessa população. Tal fato sugere a necessidade de uma vigilância em saúde e nutrição na rotina dos serviços a fim de garantir uma melhora na qualidade de vida desses idosos.

\section{REFERÊNCIAS}

1. Mendes ACG, Mendes ACG, Sá DA, Miranda GMD, Lyra TM, Tavares RAW. Assistência pública de saúde no contexto da transição demográfica brasileira: exigências atuais e futuras. Cad. Saúde Publica 2012;28(5,):955-964. doi: 10.1590/ S0102-311X2012000 500014.

2. Zattar LC, Boing AF, Giehl MWC, d'Orsi E. Prevalência e fatores associados à pressão arterial elevada, seu conhecimento e tratamento em idosos no sul do Brasil. Cad. Saúde Pública 2013;29(3):507-521. doi: 10.1590/S0102$311 \times 2013000300009$

3. Vasconcelos CMR de, Marques APO, Leal MCC, Vasconcelos EMR, Araújo EC, Raposo MCF, Vasconcelos ALR. Morbidades em idosos relacionadas ao excesso de peso. Rev enferm UFPE 2014;8(supl. 1):2228-36. doi: 10.5205/ reuol.5927-50900-1-SM.0807supl201405.

4. França VF, Pissaia E, Azzolini T, Yonemitsu EL, Giongo PL, Cervo AL, Lovato ECW, Schiavoni D. Estado nutricional e condições de saúde de idosos de Francisco Beltrão, Paraná. Nutrire 201540(3):337-343. doi: 10.4322/2316-7874.56214.

5. Christmann AC, Zanelatto C, Semchechem CC, Novello D, Schiessel, DL. Perfil de risco de doenças cardiovasculares e estado nutricional de idosos ativos de Guarapuava - Paraná. UNOPAR Cient Ciênc Biol Saúde 2013;15(ESP):349-56.

6. Silva RA, Jacinto PVO, Lopes ACS, Santos LC. Perfil de nutrição e saúde de usuários da atenção primária a saúde. Revista Baiana de Enfermagem 2011;25(3):287-299.

7. OPAS. Organização Pan-Americana .XXXVI Reunión del Comitê Asesor de Ivestigaciones en Salud - Encuestra Multicêntrica - Salud Beinestar y Envejecimeiento (SABE) en América Latina e el Caribe - Informe preliminar

8. IBGE. Projeção da população do Brasil e das Unidades da Federação. 2015. Disponível em: <http://www.ibge.gov.br/ apps/populacao/projecao/index.html>.

9. Leite CC, Rodrigues-Gonçalves MC, Rios-Asciutti LS, LeiteCavalcanti A. Prevalência de doenças crônicas e estado nutricional em um grupo de idosos brasileiros. Rev. salud pública 2009;11(6):865-877.

10. Bassler TC, Lei DLM. Diagnóstico e monitoramento da situação nutricional da população idosa em município da região metropolitana de Curitiba (PR). Rev. Nutr 2008;21(3):311321. doi: 10.1590/S1415-52732008000300006.

11. Alves LC, Leimann BCQ, Vasconcelos MEL, Carvalho MS, Vasconcelos AGG, Fonseca TCO. A influência das doenças crônicas na capacidade funcional dos idosos do Município de São Paulo, Brasil. Cad. Saúde Pública 2007;23(8):19241930. doi: 10.1590/S0102-311X2007000800019.

12. Scherer R, Scherer F, Conde SR, Dal Bosco SM. Estado nutricional e prevalência de doenças crônicas em idosos de um município do interior do Rio Grande do Sul. Rev. bras. geriatr. gerontol 2013;16(4):769-779. doi: 10.1590/S180998232013000400011.

13. Nascimento CM, Ribeiro AQ, Cotta RMM, Acurcio FA, Peixoto SV, Priore SE, Franceschini SCC. Estado nutricional e fatores associados em idosos do Município de Viçosa, Minas Gerais, Brasil. Cad. Saúde Pública 2011;27(12):2409-2418. doi: 10.1590/S0102-311X2011001200012.

14. Kümpel DA, Sodré AC, Pomatti DM, Scortegagna HM, Filippi J, Portella MR. Obesidade em idosos acompanhados pela estratégia de saúde da família. Texto contexto - enferm 2011;20(3):471477. doi: 10.1590/S0104-07072011000300007.

15. Geib, Lorena Teresinha Consalter. Determinantes sociais da saúde do idoso. Ciência \& Saúde Coletiva 2012;17(1):123-133.

16. Victor JF, Ximenes LB, Almeida PC, Vasconcelos FF. Perfil sociodemográfico e clìnico de idosos atendidos em Unidade Básica de Saúde da Família. Acta Paul Enferm 2009;22(1):49-54.

Como citar: SANTOS, Bianca Bernhard dos; COUTO, Analie Nunes; WICHMANN, Francisca Maria Assmann. Estado nutricional de idosos atendidos na Atenção Primária à Saúde do município de Candelária/RS. Cinergis, Santa Cruz do Sul, v. 17, out. 2016. ISSN 2177-4005. Disponível em: <https://online.unisc.br/seer/index.php/cinergis/artic/e/view/8155>. Acesso em: 11 out. 2016. doi:http://dx.doi.org/10.17058/cinergis.v17i0.8155. 